\title{
Ueber den Einfluss des Lichtes auf den Thierkörper.
}

Von

\section{H. Quincke}

in Kiel.

Bekannt, wenn auch zur Zeit wenig gewürdigt, ist der Einfluss des Lichtes auf das Gedeihen des Menschen.

Das lebhaftere und aufgewecktere Wesen des Südländers mag nicht minder von dem reichlicheren Lichtgenuss, wie von der Erleichterung aller Existenzbedingungen durch den höheren Sonnenstand abhängen. In Gebirgsthälern hat der Instinkt von Generationen für die menschlichen Ansiedelungen die sonnenbesehienensten Punkte mit Vorliebe wählen lassen und die ärztliche Erfahrung zeigt, dass sonnige Wohnungen und Wohnstätten der Gesundheit vortheilhafter sind als sonnenarme. Vielerlei Umstände dürften diesen Vorzügen zu Grunde liegen: die Wärne und Trockenheit des Bodens und des Hauses, daher reinere Luft; - schlechteres Gedeihen der Mikroorganismen, - bessere Conservirung der Nahrungsmittel; - bei zerstreut liegenden Wohnstätten besseres Gedeihen der Vegetation und damit bessere Existenzbedingungen überhaupt. Die Verhältnisse liegen zu complicirt, um zu entscheiden, wie weit hier eine Einwirkung des Lichtes auf den Menschen selbst etwa mitspielt.

Dass das Licht als Lebensreiz wirkt, zeigt sich bei vielen Menschen in dem Einfluss des Wetters auf die Stimmung; sie ist bei trübem Himmel und in den kurzen Wintertagen deprimirt, während helles Wetter Energie und Lebenslust steigert. Besonders deutlich zeigt sich dies an den ersten hellen Frïlingstagen. Ihr anregender Einfluss hat dann meist grössere Ermüdung zur Folge, die, wie ich glaube mit Unrecht, gewöhnlich allein der - oft nur geringfügigen - Steigerung der Lufttemperatur zugeschrieben wird. Dass bei Hochgebirgswanderungen das Abnebmen der Schneebrille momentan das Ermiudungsgefühl beseitigt, während das andauernde intensive Licht der Schneefelder die Ermüdung steigert, ist eine wohl vielfach gemachte Erfahrung, die vor kurzem noch L o e b (1. e. S. 100) ausgesprochen hat. 
Ich selbst glaube mit Bestimmtheit behaupten zu können, dass Licht, namentlich Sonnenlicht von günstigem Einfluss auf das körperliche Gedeihen vieler chronisch Kranker und Reconvalescenten, ja auf den schliesslichen Verlauf mancher fieberhafter Krankheiten, wie Typhus und Pneumonie ist. Klima und sonstige Umstände bedingen es, dass eine schädliche Wirkung zu vielen Lichtes auf Kranke uns viel seltener zur Wahrnehmung kommt.

Auch unsere Hausthiere (Hunde, Katzen, Hühner) sehen wir die Sonne suchen und ihre Strahlen der Ofenwärme vorziehen. "Sonnenbäder" - in Luft und Wasser - bilden hie und da noch einen Bestandtheil des Heilschatzes der Volksmedicin.

Die Wirkung des Lichtes in hygienischer und therapeutischer Beziehung ist in den Zeiten der reinen Empirie wahrscheinlich mehr anerkannt und praktisch verwerthet worden, als heutzutage, wo sie von Aerzten, Baumeistern und Laien unterschätzt und bei der Behandlung von Kranken wie bei der Anlage von Wohnungen nicht genügend beriicksichtigt wird. Der Grund dafür liegt in dem sonst schätzbaren Streben nach Exactheit. Denn wohl ist für zahlreiche chemische Vorgänge der Einfluss des Lichtes genau bekannt und auch vielfach techniseh verwerthet, wohl ist die handgreifliche Wirkung des Lichtes auf das Gedeihen der Pflanzen eingehend untersucht und seine Beziehung zu Oxydation, Reductionen und Chlorophyllbildung sogar unter Berücksichtigung der einzelnen Abschnitte des Spectrums erforscht; recht spärlich aber sind in der That die wissenschaftlichen Beweise für die Wirkung des Lichtes auf den Thierkörper in der einen oder anderen Richtung; denu die Deutung der Thatsachen ist schwierig, weil die Lichtwirkung gewöhnlich durch eine Reihe anderer Umstände complicirt wird und namentlich, weil Wärme und Licht schwer von einander zu trennen sind. -

Die Wirkung des Lichtes auf den Thierkörper kann zu Stande kommen :

1. durch den direkten Einfluss auf die Zellen selbst,

2. durch Erregung centripetaler Nerven und dadurch ausgelöste Reflexe.

Der direkten Beeinflussung zugänglich ist, soweit wir nicht künstlich durch Kleidung das Licht abschwächen oder absperren, vorwiegend die äussere Haut; die künstlichen Beund Durchleuchtungsversuche der Körperhöhlen, welche zu dia- 
gnostischen Zwecken angestellt wurden, zeigen aber, dass intensives Licht an vielen Stellen sicher mebrere Centimeter tief die Gewebe za durchdringen vermag (durch das Blut wird dabei allerdings eine erhebliche Abschwächung gerade der stärker brechbaren Strablen stattfinden).

Bei der Erregung centripetaler Nerven durch Licht haben wir natürlich wesentlich, aber doch nicht mit Nothwendig. keit ausschliesslich an den $\mathrm{N}$. opticus zu denken; denn wenn beliebige Zellen überhaupt der Lichtwirkung zugänglich sind, so ist es von vornherein wabrscheinlich, dass die Nervenendigungen der Körperdecke davon keine Ausnahme machen. Beobachtungen an angenlosen Thieren zeigen auch, dass sie in der That auf Lichtwirkung reagiren. Wie andere Reize, welche die centripetalen Nerven treffen, würde auch die photische Reizung der Hautnerven Reflexe sehr verschiedener Art in den verschiedensten Organen, namentlich im Circulations- und Respirationssystem und im Stoffwechsel auslösen können, während von Reizen, welche den N. opticus treffen, diese Functionen vorwiegend auf dem complicirten Umwege psychischer Thätigkeit beeinflusst werden.

Statt der erregenden Wirkung mässiger Lichtreize werden wir von starken unter Umständen eine lähmende zu erwarten haben.

Von den Thatsachen, welche über die Wirkung des Lichtes auf den Thierkörper bekannt sind, führe ich die hauptsächlichsten kurz an ${ }^{1}$; zunächst die über $B$ e e influssung des Stoffwe chsels: Frösche, die im Hellen gehalten werden, scheiden nach $\mathrm{Moleschott} 1 / 12$ bis $1 / 4$ mehr an $\mathrm{CO}_{2}$ aus als im Dunklen gehaltene Thiere. $P f l u ̈ g$ e $r$ und von $P l$ aten untersuchten den respiratorischen Gaswechsel an tracheotomirten Kaninchen, denen die Augen abwechselnd undurchsichtig verschlossen wurden; sie fanden im Licht die $\mathrm{CO}_{2}$-ausscheidung $=114$, die 0 -aufnahme $=116$, wenn die gleichen Functionen bei verschlossenen Augen $=100$ gesetzt werden. Moleschott und $\mathrm{Fubini}$ untersuchten die Gesammt- $\mathrm{CO}_{2}$-ausscheidung bei Frosoh, Sperling, Ratte und Haselmaus, die im Hellen und im Dunklen gehalten wurden; sie fanden

1) Ausführliche Darstellungen derselben finden sich bei Engelmann, J. Raum, F. Hammer und J. Schickhardt. 
die $\mathrm{CO}_{2}$-ansscheidung im Hellen erheblich gesteigert, im Verhältniss von 120 bis 140:100. Bei blinden Thieren betrug die Steigerung nur 111 bis $127: 100$.

Bei Fröschen wirkte das Licht auch, wenn die Centralorgaue zerstört waren. Auch an Muskeln von Frosch, Kaninchen und Hund, welche von Baut entblösst und des Kreislaufs beraubt waren, wurde die $\mathrm{CO}_{2}$-ausscheidung durch Licht gesteigert im Verbältniss von $100: 141$ bis 177 . Hirn- und Rückenmark der Säugethiere geben einen Unterschied von 100:129.

Neuerdings untersuchte Graffenberger (bei We iske) den Stoffwechsel ron Kaninchen, die theils im Hellen theils im (fast) dunklen Raum gehalten wurden. Den $\mathrm{N}$-umsatz fand er unbeeinflusst von Licht, auch den Verdauungscoëfficienten gleich; dagegen war in Dunkelheit, wenn sie nicht zu lange währte, der C-umsatz geringer, das Körpergewicht stieg, hauptsäcblich, weil mehr Fett gebildet und abgelagert wurde. Bildung und Menge des Leberglycogens wurden nicht beeinflusst. Dunkelleit bewirkte Verminderung des Hb-gehaltes, bei längerer Einwirkung auch Verminderung der Menge des Blutes.

Spieck fand bei sich selbst für die durch die Lungen ansgeathmete $\mathrm{CO}_{2}$ und den aufgenommenen 0 keine Unterschiede, ob er die "Augen mit einem Tuche verband oder ob er durch farblose, gelbe oder violette Brillen sah, doch dauerte jeder seiner Versuche höchstens 12 Min.

Die $\mathrm{CO}_{2}$-ausscheidung der Haut an Hand und Vorderarm des Mensehen fanden $\mathrm{Fubini}$ und Ronchi im Licht gesteigert. $(113: 100$.)

Marmé und Moles chott hatten schon früher berichtet, dass bei im Licht" gehaltenen Fröschen die Erregbarkeit der Nerven und Muskeln grösser und der Muskelstrom stärker sei als bei dunkel gehaltenen Thieren, doch hebt $\mathrm{Her} \mathrm{m}$ an $\mathrm{n}$ hervor, dass es sich nur um geringfïgige Unterschiede der Mittelzahlen handle.

Das Körpergewicht sah Fubini schneller abnehmen bei sehenden als bei blind gemachten Fröschen (im Verhältniss von $2,29: 1$ ).

Die Mehrzahl dieser Versuche weist auf eine Steigerung des Stoffwechsels unter dem Einfluss des Lichtes hin; dieselbe geschieht nur zum Theil durch Erregung des N. opticus.

Andere Versuche betreffen die Wirkung des Lichtes auf das 
Wachsthum der Thiere. Der Beobachtung von Edwards, dass Froschlarven sich im Dunkeln nur unvollkommen entwickelten, hatten $\mathrm{Higginbottom}$ und $\mathrm{Mac} \mathrm{Donnell}$ widersprochen S c h n e t zl e r konnte bei vollkommenerer Versuchsanordnung feststellen, dass bei Froscheiern, die in farblosen und in dunkelgrïnen Gläsern, sonst aber ganz gleich gehalten wurden, die Larven in dem dunklen Glase später ausschliupfen und sich langsamer entwickeln als in dem hellen Glase. Bei jungen Katzen constatirte $\mathrm{Hammond}$ in einem freilich etwas fragmentarisehen Versuch den retardirenden Einfluss der Dunkelheit auf das Wachsthum.

Einige Forscher suchten auch zu ergründen, ob verschiedene Farben verschieden wirksam seien; so sah Béclard Frösche im grünen Licht mehr $\mathrm{CO}_{2}$ ansscheiden, als im rothen. $\mathrm{Selmi}$ und Piacentini fanden beim Hunde, $\mathrm{P}$ ot t bei der Maus die $\mathrm{CO}_{2}$-ausscheidung in absteigender Reihe beeinflusst durch gelbes, grünes, blaues, weisses (!), rothes und violettes Licht, Mole se h ot t und $\mathrm{Fub}$ in $\mathrm{i}$ sahen dagegen die $\mathrm{CO}_{2}$-ausscheidung bei allen Thieren im violetten Licht ebenso stark wie im weissen, während roth bei Fröschen wie Dunkelheit und nur bei Säugethieren steigernd wirkte. Auf blinde Säugethiere wirkte das farbige Licht weniger steigernd als auf unversehrte. Fliegeneier und Larven sah B é c 1 a r d unter violetten und blauen Glasglocken sich besser entwickeln als unter rothen, gelben und weissen; am schlechtesten gediehen sie im grünen Licht.

Die Entwicklung der Eier von Frosch, Forelle und Lymnaea stagnalis sah E. Young im Dunkeln verzögert. Violettes, blaues, gelbes und weisses Licht wirkten in absteigender, Reihe fördernd, während rothes und grünes Licht die Entwicklung gänzlich sistirte, also schädlich zu wirken schien.

Da Kaulquappen ohne Nahrung im violetten und blauen Lichte schneller starben, als in andersfarbigem, schloss $\mathrm{Y}_{0} \mathrm{a} \mathrm{ng}$ auf Erhöhung des Stoffweehsels in jenem.

Wie man sieht, enthalten diese Versuche mit farbigem Licht manche Widersprüche in sich, wenn anch im Ganzen die grössere Wirksamkeit der blauen und violetten Strahlen daraus bervorgeht; auffällig ist nur, dass diese wirksamer sein sollen, als weisses Licht. Auch ist bei dem farbigen Licht die Lichtintensität nicht berücksichtigt resp. nicht vergleichbar gewesen und ist die Farbe der Gläser nur ausnahmsweise spectroscopisch analysirt. 
Ueber die Wirkung des Lichts a uf einzelne Zellen und Gewe be weiss man Folgendes: Das Protoplasma farbloser Blutkörper und anderer amöboider Zellen scheint nach En ge 1m a n n, was Contractilität betrifft, vom Licht unabhängig zu sein.

Nach Usk of zeigen Leukocyten des Frosehes im rothen Licht zahlreichere und längere Fortsätze als im violetten.

Die Flimmerbewegung wird nach Engelman nom Licht nicht beeinflusst; nach $U \mathrm{sk}$ of $\mathrm{f}$ soll sie bei rothem und violettem Licht gleich schnell geschehen, aber für einen Augenblick stehen bleiben, wenn man das violette Licht durch rothes ersetzt.

A uerbach sah bei der Furchung der Froscheier, dass Tagesund Sonnenlicht ein energischer Reiz für die Contractionen des Eiprotoplasmas ist und durch diese bei künstlicher Lageänderung des Eies das Pigment stets nach der belichteten Seite desselben hinübergeschoben wird. Jakimowits $\mathrm{ch}$ fand bei Triton cristatus, dass die Energie der Zellenvermehrung direkt von verschiedenen farbigen Reizen abhängig ist.

Von specifischen Zellen zeigen nach Engelmann die Pigmentzellen und Zapfen der Retina eine Ortsveränderung, ein Herabsteigen auf Lichteinfall.

Die Iris contrahirt sich am ausgeschnittenen Auge von Amphibien und Fröschen auf Lichteinfall auch nach Entfernung der Retina; es ist nicht sicher entschieden, ob dies unter Mitwirkung von Ganglienzellen oder durch direkte photische Reizung der glatten Muskelfasern geschieht.

Die Pigmentzellen der Hant werden bei Thieren vom Licht sehr deutlich zu Bewegungen veranlasst, aber in verschiedenem Sinne: während sie beim Chamäleon im Licht sich ausbreiten und die Haut dunkler färben, also eine Art Schutzvorrichtung, wird bei Fröschen und Fischen die Haut im Licht heller durch Contraction der Pigmentzellen, also eine Art von Anpassung.

Die Farbänderung geschieht nach Engelmann (l. c. S. 370) angeblich meist als Reflex rom Auge aus; beim Chamäleon stebt sie nach P. B ert zwar ebenfalls unter dem Einfluss des Centralnervensystems, findet aber auch noch einige Zeit nach dem Tode, also wohl auch durch direkten Lichtreiz statt.

Beim Menschen (kankasischer Raçe) wird unter dem Einfluss des Sonnenlichtes die Haut bekanntlich pigmentreicher, gewöhnlich in diffuser Weise, seltener in Gestalt kleiner Flecke, der Sommer- 
sprossen. Nar bei sehr intensiver Einwirkang bewirkt die Sonne acute Hautentziindung mit Abschilferung der Epidermis. Wenn auch für das Zustandekommen dieser Veränderungen oft Hilfsmomente mitwirken, wie Trockenheit, Kälte und Bewegung der Luft, and wenn.auch die individuelle Anlage dabei eine grosse Rolle spielt, so weist die unbefangene Beobachtung doch mit grosser Sicherheit auf das Licht als hauptsächliche Ursache hin. Durch Beobachtungen und Versuche von Veiel, Maklak of f, Widmark und Hammer ist nun mit Sicherheit erwiesen, dass die Wärmestrahlen an dieser Wirkung auf die Haut nicht betheiligt sind, dass sie vielmehr wesentlich von den stärker brechbaren Strahlen des Spectrums, namentlich den ultravioletten, abhängt; das an letzteren reiche elektrische Bogenlicht wirkt sehr stark auf die Haut. Wäbrend die durch Wärme erzeugte Hautröthe schnell entsteht und schnell verschwindet, erfolgt durch Licht die Röthung gewöhnlich erst nach einigen Stunden, ist aber anhaltender und von Ablösung der Oberhaut und Pigmentablagerung gefolgt. Letztere kann als Schutzmittel gegenüber der Lichtwirkung gedeutet werden. Wie bei anderen Reizen spielt auch beim licht Gewöhnung der Haut eine Rolle, daher im Frïhjahr und bei Städtern die acuten Folgen leichter eintreten. Ob die Lichtwirkung auf die Haut direkt oder durch Vermittelnng der Nerven zu Stande kommt, vermögen wir vorläufig nicht zu entscheiden; wahrscheinlicher ist mir ersteres.

Von Lichtwirkungen auf den Chemismus isolirter Organe kennen wir die oben erwähnte Steigerang der $\mathrm{CO}_{2}$-ausscheidung der Muskeln und des Centralnervensystems (Molesehott und Fubini) sowie die Zerstörung des Sehroths in der Retina. Hierher gehört auch die Beobachtung von Buchner, dass durch Tageslicht und noch mehr durch Sonnenlicht die globulicide und keimtödtende Wirkung des Blutserums vernichtet wird; da diese Wirkung im O-leeren Raume geringer ist, scheint das Licht wenigstens theilweise durch Anregung von Oxydation zu wirken.

Ausserordentlich zahlreich sind die Beobachtungen über d as Verbalten der Thiere gegenüber dem Licht und den verschiedenen Farben und ihre Deutung ist im Allgemeinen um so schwieriger, je höher organisirt das Thier. Für die von uns angestellten allgemeinen Betrachtungen sind von Wichtigkeit die Beobachtungen von V. Graber, dass auch augen- 
lose Thiere z. B. Regenwiirmer oder der Augen beraubte Thiere (Tritonen und Schaben) durch die Wahl ihres Aufenthaltsortes eine Scheu vor hellem Licht und speciell vor den stärker brechbaren Strah̆len des Spectrums, also ein Unterscheidungsvermögen nicht nur für Licht, sondern auch für Farben zeigen. Ob hier nur die Nervenenden der Körperoberfläche oder auch andere Gewebselemente lichtempfindlich sind, kann nicht entschieden werden.

Möglich, dass solche von den Augen unabbängigen Lichtwirkungen auf den Körper auch bei dem von $\mathrm{L}$ o eb für viele Thiere nachgewiesenen Heliotropismus mitspielen.

Dass auch Lebewesen einfachster Organisation auf Licht reagiren, zeigen die Beobachtungen von E $\mathrm{n}$ ge e lma n n (Bewegungsstillstand von Pelomyxa palustris bei plötzlicher Beleuchtung Bewegung des Loheplasmodium Aethalium zur Oberfläche im Dunkeln, in die Tiefe bei Hellem - Vorliebe des Bacterium photometricum für Licht und für gewisse Regionen des Spectrums Lichtbedürfniss der Englena viridis mit besonderer Lichtempfindlichkeit ihres oralen Körperendes.

Für die Aerzte von besonderem Interesse ist die schädliche resp. vernichtende Wirkung intensiven Lichtes auf viele, namentlich auf pathogene Bacterien. Freilich ist es von vornherein wahrscheinlich, dass wie bei den Thieren so auch bei den Bacterien sehr verschiedene Grade der Lichtempfindlichkeit no Lichtfreundlichkeit vorkommen dürften und dass die Grenze zwischen wohlthätig anregender und lähmender oder vernichtender Wirkung des Lichtes für verschiedene höhere wie niedre Organismen bei sehr verschiedenen lichtintensitäten zu suchen sein wird. -

Ich habe nun Beobachtungen gemacht, welche in sehr einfacher Weise, zunächst freilich nur qualitativ, darthun, dass durch Licht die Oxydation in thierischen Zellen gesteigert wird. Der Nachweis geschieht durch die Farbenanderung, welche Blut oder Bismuthum subnitricum dabei erleiden.

Am Einfachsten gestaltet sich der Versuch mit Eiter; dieser wird mit frischem geschlagenen Blut $\left(1 / 20\right.$ bis $1 / 10^{\circ}$ seines Volumens) oder mit einigen Tropfen einer wässrigen Suspension von Bismuthum subnitricum versetzt und durchgeschiittelt. Mit dem Gemisch werden Reagenzgläser, einige Centimeter hoch gefuillt, am Fenster der Sonne oder dem Tageslicht ausgesetzt. Dünnere 
Schichten kann man durch Einsenken eines etwas beschwerten dünneren Reagenzglases in das erstere herstellen, oder noch zweckmässiger so, dass man einige Tropfen des Gemisches in ein flaches Uhrglas giesst und in dieses, ebenfalls mit der convexen Seite nach unten, ein zweites Uhrglas von etwas kleinerem Durchmesser und etwas geringerem Krümmungsradius setzt, sodass sich eine in der Mitte etwas dïnnere Flüssigkeitsschicht von einer Dicke bis etwa $1 \mathrm{~mm}$ bildet. Den Flüssigkeitsrand kann man durch einige Tropfen Paraffinum liquidum von der Luft abschliessen. Beliebig. dünne Flüssigkeitsschichten stellt man in Form gewöhnlicher mikroscopischer Präparate durch Unterschieben von Deckglas. splittern her; hier ist ein Rand von festem Paraffin zur Verhütung des Eintroeknens zweckmässig.

Solche mit dem Flüssigkeitsgemisch beschickte Gläser werden stets paarweise hergestellt, das eine in einem völlig dunklen Kasten aufbewahrt, das andere - in einem Reagenzglasgestell, auf einem weissen Porzellanteller oder Papier - am offenen Fenster dem Licht ausgesetzt.

An frisch entnommenen und zerkleinerten Organen hat P. Bert, wie schon früher Spallanzani, gasanalytische Versuche über O-zehrung und $\mathrm{CO}_{2}$-produktion gemacht und dieselbe nicht ganz unbedeutend gefunden. An dem fliussigen Gewebe, dem Eiter, kann man diese 0 -zehrung bei der Eröffnung von Abscessen deutlich erkennen, indem mitergossenes hellrothes Blut sehr schnell venöse Farbe annimmt; schlittelt man solchen Eiter im Reagenzglase mit Laft, so stellen sich die hellrothe Farbe und (im auffallenden Licht spectroscopisch betrachtet) die $\mathrm{OHb}$-streifen wieder her, um nach kurzer Zeit der blaurothen Farbe und dem einfachen $\mathrm{Hb}$-streifen Platz zu machen. Bei weiterem wiederholtem Schütteln danert es “immer länger bis der .OHb-streif versehwindet; diese Verzögerung zeigt sich auch, wenn man frische, bisher noch nicht benutzte Portionen Eiter zu versehiedenen Zeiten dem:Versuch unterwirft. Die Reductionsenergie des Eiters nimmt.also nach der Entleerung rasch ab.

Diese auch im Dunkeln nachweisbaren Oxydationsvorgänge im Eiter werden nun durch Tageslicht, noch mehr durch Sonnenlicht sehr erheblich gesteigert. Ein Beispiel giebt: 


\section{Versuch I.}

3. J u li 93. Acute eitrige Pleuritis. Incision 1 Uhr. Von dem mit wenig Blut gemischten Eiter wurden $1 \frac{1}{2}$ Uhr zwei Proben in Reagenzgläsern mit Luft geschüttelt und senkrecht aufgestellt
a. In der Sonne.
b. Dunkel.

5 Min. später: Der $\mathrm{OHb}$ streif ist Der OHb streif noch schwach erhalten. verschwunden.

Ein zweites Gläserpaar mit etwas blutreicherem Eiter;

Nach 5 Min. Auf der dem Fenster Etwas Verfärbung; OHbstreif noch zugewendeten Glasseite vollkom- erhalten.

men venöse Färbung, deutlich verschieden von der auf der

Schattseite des Glases.

Nach $10 \mathrm{Min.} \quad$ Doppelstreif verschwunden.

A bends 7 Uhr: (Sehr mässiges diffuses Himmelslicht). Durch Schütteln mit Luft wird die OHb-Farbe wieder hergestellt; erst nach 1/2 Stunde ist er verschwunden, im dunkel, wie im hell gehaltenen Glase.

4. Juli, y1/2 Ubr. Von dem noch nicht benutzten Eiter wird einem Theil 5 resp. 10 Vol. ${ }^{\circ} / 0$ geschlagenen Schröpfkopfblutes beigemischt; die Mischung in Reagenzgläsern aufgestellt:
a. Diffuses Himmelslicht.
b. Dunkel.

$10 \mathrm{Uhr}$. In keinem der Gläser ist Farbenänderung erkennbar; der $\mathrm{OHb}$. streif ist sichtbar.

$10^{3} / 4$ Uhr. Im $5 \%$ Glase ist der OHbstreif erhalten.

$\mathrm{OHb}$ streif verschwunden, im

$10 \%$ Glase abgeschwächt. Die

Gläser waren zuletzt kurze Zeit

besonnt; Sonn- und Schattseite

sind deutlich verschieden gefärbt.

(Sonnenlicht wechselt sehr.)

1 Uhr. Auch im 10\% Glase der OHbstreif verschwunden; die Schattseite noch etwas röther als die Sonnseite.

6 Uhr Abends.

Das $5 \%$ Glas völlig venös, das $10 \%$ Glas zeigt noch Spur des $\mathrm{OHb}$ streif.

Das $10 \%$ Glas noch etwas heller als das entsprechend beleuchtete; spectroscopisch kein Unterschied erkennbar.

5. Juli, $91 / 2$ Uhr. Die 4 Gläser werden mit Luft geschüttelt und werden hellroth.

(Wechselndes Sonnenlicht.) 
$11 \frac{1}{2}$ Uhr. Das 5 und $10 \%$ Glas Das 5 wie $10 \%$ Glas sind zwar etwas sind dunkel gefärbt, zeigen keinen dunkler geworden, zeigen aber beide Doppelstreif mehr. noch den Doppelstreif.

22. J u li, $1 \mathrm{Uhr}$. Von dem nunmehr 19 Tage alten (aber noch geruchlosen) Eiter wird ein Theil mit frischem Schröpfkopfblut (nach Schätzung $5-10 \%$ gemischt. Das Wetter ist hell, theilweise Sonnenschein.

4 U hr. Venöse Färbung, anf Sonn- Noch hellrothe Färbung.

seite stärker als auf Schatt-

seite.

23. Juli, 9 Uhr früh. Beide Gläser venös gefärbt.

Es ergiebt sich also, dass die Intensität der 0.Zehrung im Licht viel intensiver ist als im Dunkeln, dass sie für beide Fälle unmittelbar nach der Entleerung des Eiters am stärksten ist und vom ersten zum zweiten, sowie vom zweiten zum dritten Tage abnimmt; nach 20 Tagen scheint eine weitere geringe Abrahme stattgefunden zu haben.

\section{Versuch II.}

24. März 1894. Eiter soeben aus Drüsenabscess am Halse entleert, mit etwas Blut vermischt, wird durch Reiben im Mörser möglichst mit 0 gesättigt, dann 2 Uhrglaspräparate hergestellt; diese mit Vaselinöl abgeschlossen.
a. Helle Sonne.
b. Dunkel.

Nach 10 Min. Deutlicher Farbenunterschied.

$\mathrm{OHb}$ streif undeutlich. OHbstreif noch deutlich.

$17 \mathrm{Min}$. Venös gefärbter $\mathrm{Hb}$ streif. $\mathrm{OHb}$ streif noch deutlich. $11 / 2$ Std. Beide Gläser venös.

\section{Versuch III.}

14. Xai 1891. Dickeitriges Pleuraexsudat, vor 1 Stde. entleert, wird mit Suspension von Bismuthum subnitricam vermischt in Reagenzgläsern aufgestellt.
a. In der Sonne.
b. Dunkel.

Schon nach 10 Min.beginnende Schwärzung auf der Lichtseite des Glases, die bis zum nächsten Tage erheblich zunimmt, wegen Senkung des Bipulvers in der Nähe des Bodens am stärksten ist. Auf der Zimmerseite, wo nur diffuses Licht einwirkt, beginnt die Graufärbung erst nach 6-12 Stunden.

14 Tage später wird der Versuch mit demselben Eiter wiederholt, der bedeckt aufbewahrt wurde und noch nicht faulig riecht. 
Selbst in der Sonne ist nach mehreren Die dunkel grehaltenen Controlgläser Stunden die Mischung nur leicht grau, bleiben Tage lang weiss und schwärzen schwärzt sich aber nach mehrtägigem sich nachträglich am Licht. Stehen im Hellen.

Gegenüber dem Bismuithum subnitricum verhält sich der Eiter also etwas anders als gegenüber dem 0xyhämoglobin; während er diesem den 0 auch im Dunkeln entzieht, nur langsamer, bleibt der fester gebundene 0 des Wismuthsalzes im Dunkeln unbeeinflusst und wird a ssehliesslich im Lichte entzogen. Auch das Wismuthsalz wird von dem frischen Eiter viel schneller reducirt als einige Zeit nach der Entleerung.

Die gleichen Versuche wurden mit Blut wie mit Wismuthsalz mit einigen Variationen vielfach wiederholt und ergaben stets das gleiche Resultat. Auch in Uhrgläsern und auf Objectträgern wurden sie angestellt. An letzteren konnte man sich mikroscopisch uiberzengen, dass die Krystalle des Wismuthsalzes in der Form unverändert bleiben und sich nur an der Oberfläche schwärzten. Begreiflicherweise konnte bei geringeren Graden der Reduction die Verfärbung der Krystalle mikroskopisch noch unerkennbar bleiben, wo für das blosse Auge die Mischung schon Graufärbung zeigte.

Die Schnelligkeit der Reduction hing bei Blut wie bei Wismuthsalz ausser von der Frische des Eiters von der Li e h tintensität ab; natürlich tritt dies bei den Wismuthversuchen, wo die Reduction allein bei Gegenwart von Licht stattfindet, schlagender hervor: was bei Sonnenlicht in wenigen Minuten geschah, konnte bei diffusem Tageslicht Stunden dauern; ein heller Tag und Sommerszeit wirken stärker als ein trüber Tag oder Winterszeit. Quantitative Messungen der Lichtstärke habe ich nicht anstellen können.

Bei den Wismuthversuchen erkennt man, dass die Lichtwirkung nur in den oberflächlichsten Flüssigkeitsschichten stattfindet; bei dem Uhrglasversuch ist die Unterseite stets weniger geschwärzt, wenn im Reagenzglas die. Sonnenseite intensiv schwarz war, ist der Eiter nach Umschütteln nur eben grau.

\section{Versnch IV.}

Frischer Pleuraeiter wird mit Bismuthum subnitricum gemischt in Reagenzgläsern aufgestellt. 

a. He11.
b. Dunkel.

Durch häufig wiederholtes Schütteln werden die an der Oberfläche gebildeten Oxydulschichten immer im Eiter vertheilt, so dass er nach 48 Stunden dunkelgrau gefärbt ist.

Neben diesem Glase wird nun das bis dabin dunkel verwahrte weiss gebliebene Vergleichsglas umgeschüttelt und in die 'Sonne gestellt. Nach 2 Stunden ist die Sonnseite des Glases b schwärzer als die des Glases a, nach dem Scbütteln beider aber der Inhalt von b viel heller als von a. Es warenalso offenbar heute in der 0 berflächenschicht des länger belichteten Glases a nur noeh weniger reducirende Theileenthalten als in der gleichen Schichtdes unbeleuchtet gewesenen b.

Bei wiederholtem Umschütteln und Weiterbelichten in den folgenden Tagen zeigt sich ähnliches Verhalten, doch wird der Untersebied allmählich geringer und die Graufärbung beider Gläser nach dem Schütteln schliesslich gleich.

Am geeignetsten für diese Versuche erwies sich mir frischer guter Eiter von acuter Pleuritis, da er besser als Zellgewebseiter rein und in genügender Menge erhältlich war. Doch wirkte letzterer, sowie eitriges Schleimbautsecret (von der Nase, aus der Urethra, eitriges Sputum) ebenso stark reducirend. Liess man seröseitrige Exsudate absetzen, so zeigte sich, dass nur der zellreiche Bodensatz, nicht aber das klare Serum reducirend wirkten.

Oben wurde erwähnt, dass die Reductionsenergie des Eiters nach der Entleerung aus dem Körper sehnell abnimmt. Solche Abnahme erfolgt auch im Körper, wenn der Eiter längere Zeit in Höhlen verweilt, und seine Zellen sich dabei verändern, körnig zerfallen und die Kerne undeutlich werden oder schwinden.

\section{Versuch V.}

17. Juli 1893. Eiter aus einem tuberkulösen Senkungsabscess der Wirbelsäule bei der Section erhalten, wird mit Bismuthum subnitricum gemischt in Reagenzglas und Uhrglas aufgestellt. Nach 20 Stunden (diffuses Tageslicht, theilweise Sonne, kurze Nacht) zeigen die belichteten Gläser nur geringe Graufärbung, die in den nächsten Tagen langsam zunimmt.

Aehnlich verhält sich Eiter aus einem anderen Wirbelsäulenabscess, sowie Eiter, welcher operativ aus tuberculösen Abscessen entleert war.

Die käsige Masse aus einer frisch exstirpirten scrophulösen Lymphdrüse mit Wismuth zerrieben, zwischen Uhrgläsern, färbt sich in der Sonne nach $1 / 2$ Stunde grau, wird auch nach mehrtägigem Stehen nicht schwarz. 


\section{Den Einfluss des Kochens zeigt}

\section{Versuch VI.}

7. Ju li. Pleuritiseiter, 3 Tage alt, wird mit Bismuthum subnitricum gemischt in 4 Reagenzgläser gefüllt und zwei davon 1/4 Stunde lang im Wasserbade gekocht. 2 Gläser, ein gekochtes und ein ungekochtes werden nun der Sonne exponirt, die beiden andern zur Controle im Dunkeln verwahrt. Letztere bleiben unverändert; von den belichteten Gläsern zeigt das gekochte in der ersten Viertelstunde stärkere Schwärzung als das ungekochte nach 2 Stunden aber ist letzteres dunkler und bleibt anch bei wiederholtem Umschütteln und Belichten dunkler als das gekochte.

Von dem gleichen Wismutheitergemisch wurden, sowohl von der rohen, wie von der gekochten Portion, Uhrglaspräparate hergestellt. In dem gekochten Präparate war nach 15 Min. die Schwärzung schon intensiver als im rohen; nach 1 Stunde war letzteres aber dunkler und blieb es auch, als beide Gläser noch weiter belichtet wurden und nachdunkelten.

Man wird dies. so deuten müssen, dass in dem gekochten Fiter die reducirbare Substanz für die Lichtwirkung zwar leichter angreifbar, aber in geringerer Menge vorhanden war als im ungekochten.

Während lebende resp. überlebende Gewebe dem Blute energisch Sauerstoff entziehen, findet im arteriellen Blute selbst bekanntlich nur eine äusserst geringfügige 0 -zehrung statt, sodass dieses, ausserhalb des Körpers aufbewahrt, Tage lang seine hellrothe Farbe bewaint. Ganz anders das $\mathrm{le} \mathrm{uka} \in \mathrm{m}$ is $\mathrm{e} \mathrm{he} \mathrm{B} l \mathrm{ut}$ ! Dieses, durch Aderlass gewonnen und in Gläsern aufbewahrt, zeigt $\left.^{1}\right)$ schon nach kurzer Zeit (10-15 Minuten) den Umschlag in die venöse Farbe; offenbar durch 0-zebrung seitens der farblosen Elemente. Wie beim Gemisch von Blut und Eiter lässt sich auch vom leukaemischen Blut durch wiederholtes Schütteln mit Luft nachweisen, dass diese O-zehrung länger als 24 Stunden andauert, mit allmählich abnehmender Energie.

In mehreren Fällen konnte ich diese Farbenänderung des leukaemischen Blutes - mit blossem Ange und spectroscopisch auch erkennen, wenn ich nur den durch Fingerstich gewonnenen Blutstropfen in einem Capillarrohr auffing oder in einem etwas dicken Deckglaspräparat mit Paraffin oder Vaselinöl abschloss; sie vollzog sich in einem Fall in einer balben Stunde, in anderen erst nach mehreren Stunden, je nach dem Grade der Leukämie aber

1) Vgl. meine Mittheilung auf der Naturforscher-Versammlung zu Heidelberg 1889. 
anch nach verschiedenen Umständen bei der Herstellung des Präparats.

In 3 verschiedenen Versuchen (von 2 Fällen) wurden ein oder mehrere Präparate auf weisser Unterlage der Sonne ausgesetzt, während die Controllpräparate sich daneben unter einem schwarzen Pappkasten oder im Tischkasten befanden. Jedesmal trat die Verfärbung in der Sonne schneller als im Dunkeln ein. In dem einen Falle nicht sehr hochgradiger Leukämie war die 0zehrung im Dunkeln so gering, dass die Controllpräparate hellroth blieben; als dieselben nach drei Tagen dem Licht und der Sonne exponirt wurden, fand selbst nach 8 Stunden nur eine sehr geringe Farbenänderung statt, - während sie an den frischen Präparaten bei etwa gleich intensiver Belenchtung die Verfärbung schon nach 2 Stunden deutlich und nach 6 Stunden sebr ausgesprochen war. Wurde vor der Herstellung der Deckglaspräparate dem Blutstropfen etwas Bismuthum subnitricum beigemengt, so zeigte das in der Sonne liegende Präparat nach einer Stunde deutliche Graufärbung gegenuiber dem dunkel aufbewahrten Controllpräparate.

Einige Male zeigten Präparate leukämischen Blutes, welche in der Sonne venöse Farben angenommen hatten, beim Liegen im Dunkeln am folgenden Tage Wiederkehr der arteriellen Farbe, ohne das 0-zutritt anzunehmen war, - eine auffällige Erscheinung, die indessen in dem unten zu erwähnenden Verhalten von Eidotter und Thymus zu Wismuth eine Analogie findet.

Wird nun ausser bei den Leukocyten auch bei anderen Zellen des Thierkörpers die Oxydation unter dem Einfluss des Lichtes gesteigert?

Zur Beantwortung dieser Frage wurden Organe von frisch getödteten Thieren (Kaninchen, Hunde) oder von menschlichen Leichen (12-24 Stunden p. m.) durch Zerschneiden, Schaben, Zerreiben im Mörser oder auf dem Reibeisen möglichst zu Brei verwandelt, dieser Brei mit Blut oder mit einer wässrigen Wismuthsuspension innig gemischt und die Mischung in Uhrglas- und Deckglaspräparaten untersucht. Es ergab sich nun für die meisten der untersuchten Organe, dass die O-zehrung derselben durch Belichtung gesteigert wird. Wie beim Eiter zeigte sich auch bei den Organen das verschiedene Verhalten gegenuiber dem Blut und gegenüber dem Wismuthsalz: während dieses im Dunkeln unverändert bleibt und nur unter dem Einfluss 
des Lichts reducirt wird, vollzieht sich die Reduction des Oxyhämoglobin schon im Dunkeln und wird durch Licht nur beschleunigt. Um letzteren Vorgang bequem zu beobachten, muss deshalb die Menge des Bluts im Verhältniss zur Masse des Organbreis richtig bemessen sein, - nicht zu wenig, damit die Reduction nicht schon vor Aufstellung der Schälchen vollendet sei, - nicht zuviel, damit der Doppelstreif des Oxyhämoglobins während der Beobachtungszeit auch wirklich ausgelöscht werde; das Volumen des zugesetzten Blutes muss demnach von $1 / 10$ bis 2 Vol. des Organbreis variiren.

Die oxydationsbefördernde Wirkung des Sonnenlichtes zeigte sich nun bei Leber, Niere, Milz, Thymus, Hoden, Muskel, Herz, Hirn, hepatisirter Lnnge, Carcinom, Eigelb.

Ich führe nur einzelne Versuchsprotokolle als Beispiel auf, um ungefähre Anhaltspunkte fuir das Mass der Reduction zu geben. Genauere Messungen sind aus verschiedenen Gründen nicht anzustellen, namentlich weil ein absolutes Mass für die Beleuchtungsintensität fehlte.

\section{Versuch VII.}

Von einem durch Verbluten getödteten Hund wird das defibrinirte Blut, mit dem. Brei der Organe vermischt, untersucht $1 / 2$ Stunde nach dem Tode: Leberbrei (1 Vol.) und Blut (1 Vol.).
a. Sonne.
b. Dunkel.

5 Min. Beginnende venöse Färbung.

7 Min. OHbstreif kaum sichtbar. OHbstreif noch deutlich.

10 Min. Doppelstreif verschwunden. Doppelstreif noch eben sichtbar.

15 Min. Beide Gläser venös gefärbt, aber noch leicht unterschieden.

27 Min. Beide Gläser gleich.

\section{Versuch VIII.}

a. Von einem (andern). verbluteten Hund werden Lieber und Blut 3 Tage im Keller aufbewahrt; dann zum Versuch benutzt, etwa 2 Vol. Leberbrei (Reibeisen) und 1 Vol. Blut.
a. Sonne.
b. Dunkel.

7 Min. Venöse Farbe; einfacher $A b$ sorptionsstreif.

Noch arterielle Farbe; der $\mathrm{OHb}$ streif etwas verwaschen.

$20 \mathrm{Min}$.

Doppelstreif fast verschwunden; Glas b noch immer heller als a.

$37 \mathrm{Min}$. Beide Gläser gleich gefärbt; nur Hb streif. 
b. Derselbe Versuch wird mit einem Stück gekochter Leber gemacht.
a. Sonne.
b. Dunkel.

23 Min. Beginnende Verfärbung. Unverändert.

Doppelstreif verwaschen.

53 Min. Venöses Aussehen; einfacher Etwas dunkler; Doppelstreif ver-

Streif. waschen.

Bisalz wird durch die rohe Leber in einigen Stunden bei Sonnenlicht geschwärzt, durch die gekochte nicht.

\section{Versuch IX.}

1 Stunde nach dem Tode. Herzmuskel (1 Vol.), Blut (2 Vol.).
a. Sonne.
b. Dunkel.

4 Min. Absorptionsstreif einfach, noch

Doppelstreif nicht verwaschen.

verwaschen.

14 Mia. Ganz venös. Einfacher Streif Doppelstreif verwaschen, aber noch deutlich. erkennbar.

$25 \mathrm{M}$ in. Beide Gläser venös, doch immer noch etwąs verschieden.

Wismuth wird durch den Muskelbrei in der Sonne nicht merklich geschwärzt.

\section{Versilch X.}

Von einem secundären Lymphdrüsen-Carcinom der Achselhöhle (vor $1 / 2$ Stunde exstirpirt) wird durch Schaben mit dem Messer der Saft ausgepresst, mit etwa $1 / 2$ Vol. Blut gemischt, zwischen Uhrgläsern aufgestellt
a. Sonne.
b. Dunkel.

10 Min. Verfärbt.

15 Min. Venös gefärbt, einfacher Noch arteriell gefärbt.

Absorptionsstreif.

$30 \mathrm{M}$ in.

Venös gefärbt, doch immer noch heller als a.

Auf Wismuthsalz wirkt der Krebssaft; nach 25 Min. beginnende Dunkelfärbung, nach.einigen. Stunden schwarz-graue Färbung.

Auch ohne exacte Messungen ergibt sich mit Sicherheit, dass die reducirende. Kraft der Organe für Blut vom Zeitpunkte des Todes ab sinkt, aber selbst nach mebreren Tagen noch erheblich ist. Die Verlangsamung zeigt sich am beleuchteten wie am dunkel gehaltenen Glase, scheint aber an letzterem dentlicher hervorzutreten.

Die reducirende Kraft der einzelnen Organe ist verschieden. Vergleicht man die 5 Organe Niere, Muskel, Leber, Hoden, Hirn, 
so scheint am stärksten die Niere zi reduciren, demnächst der Muskel (Herz nnd Extremität) und die Reductionsenergie dann in der genannten Reihenfolge abzunehmen.

Der fördernde Einfluss des Lichts zeigt sich bei allen, ist aber bei langsamem Ablauf der Reduction natïrlich bequemer zu beobachten.

Für die Milz ist wegen des verschiedenen, aber immer grossen Blutgehalts der Vergleich mit anderen Organen unsicher.

Recht deutlicb (und im Licht schneller) reducirend wirkt auch die Thymusdritise rom Kalb, die vom Schlacbter bezogen und nicht friuher als 12 bis 24 Stunden nach dem Tode untersncht werden konnte. Ein sehr geeignetes, weil leicht erhältliches Untersuchungsobject ist der Dotter des Hühnereies; wird ein solcher (am zweckmässigsten sind recht blasse Dotter zu verwenden) mit 1 bis $2 \mathrm{ccm}$ Blat versetzt (etwa $1 / 7-1 / 15$ Vol.), so zeigt sich am "besonnten Uhrglaspräpa rate, sowie auf der besonnten Seite des Reagenzglases mach einer Stunde deutliche Verfärbung und nach einigen Stunden völlige Reduction des Oxyhämoglobins, während diese in den dunkel gehaltenen Vergleichsgläsern viel langsamer auftritt und oft nach 24 Stunden noch nicht vollendet

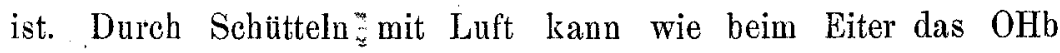
wiederhergestellt und der Versuch dann wiederholt werden.

$\mathrm{Zu}$ bemerken ist, dass verschiedene Dotter eine recht verschiedene Reductionskraft zeigen, so dass die dunkel aufbewahrten Gläser manchmal länger als 24 Stunden ihre arterielle Farbe bewahren können, während bei anderen nach einmal eingetretener Reduction das durch I.uftschütteln neu oxydirte Hämoglobin nun auch im Dunkeln schneller reducirt wird, als das erste Mal.

Nicht so auffällig wie beim Eidotter, aber doch zu bemerken war die individuelle Verschiedenheit der Reduktionsenergie übrigens auch bei manchen Organen, namentlich der Leber und der Thymusdrüse.

Das Bismuth um subuitricum, das den 0 ja sebr viel fester gebunden enthält als das $\mathrm{OHb}$, wird durch die thieriseben Gewebe auch sehr viel langsamer und ausschliesslich unter der Einwirkung des Lichtes redueirt. Nur bei den krïftigst wirkenden Organen wird die Verfärbung schon in der ersten halben Stunde deutlich; sie nimmt noch bis zur 6 . Stunde und länger zn.

Manche Organe verhalten sich in ihrer Reductionsenergie gegenüber dem Wismutbsalz ähnlich wie gegenïber dem $\mathrm{OHb}$ 
(z. B. Leber, Niere, Thymus, Hoden, Hirn), and ere zeigen ein abweichendes Verhalten, so wirkt der Muskel stark auf das $\mathrm{OHb}$ und fast garnicht auf das Wismuthsalz, während umgekehrt dureh Eigelb das $\mathrm{OHb}$ nur langsam, das Wismuthsalz aber sehr schnell und kräftig reducirt wird. Man wird daraus schliessen dürfen, dass die 0 zehrenden chemischen Vorgänge in verschiedenen $\mathrm{Or}^{\mathrm{r}}$ ganen in verschiedenem Masse betheiligt sind.

In den dunkel gebaltenen Controlpräparaten bleibt, wie schon erwähnt, das Wismuthsali selbst Tage lang vollkommen weiss, werden sie dann nachträglich der Sonne exponirt, so schwärzen sie sich.

Wurden die durch Licht geschwärzten Wismuthpräparate der Thymus oder des Eidotters ins Dunkle gestellt, so hellten sie sich nach Ablauf von einem oder mehreren Tagen merklich anf, um bei erneuter Belichtung sich wieder zu schwärzen. Der im Licht stattgefundene Reductionsprocess des Wismuths scheint also im Dunkeln wieder ruickgängig zu werden. Damit mag es zusammenhängen, dass bei sehr tribem Winterwetter eine Eigelbwismuthmischung sich selbst nach mehrtägiger Exposition nicht merklich schwärzt, die Wirkung des Lichts und der Dunkelheit halten sich hier das Gleichgewicht.

An einigen Organen (Leber, Thymus, Eidotter) wurde der E in flus s des $\mathrm{K}$ o e be $\mathrm{n}$ s untersucht:

Auf Oxyhämoglobin wirkt nach dem Kochen im Dunkeln Leber sehr langsam (vergl. Vers. VIII b), Thymus und Eigelb nicht merklich. Bei Sonnenlicht wirken alle drei Organe viel langsamer und unvollkommener als im frischen Zustande.

Wismuthsalz wird im Dunkeln von keinem der gekochten Organe reducirt; auch im Sonnenlicht wirkt die gekochte Leber nicht, während gekochtes Eigelb mindestens so stark reducirt wie rohes, und gekochte Thymusdrise zwar langsamer, aber intensiver reducirt als das frische Organ.

Alkoholgehärtete L eber ist für Wismuthsalz in der Sonne unwirksam.

Weiter wurden noch verschiedene feste und flüssige Substan. zen untersucht:

Hydroceleserum, Blutserum, Eiterserum schwärzen Wismuth im Licht nicht (wohl schwärzt sich die Oberfläche des am Boden gesammelten Bismuthbreis, wenn das Serum zellige Elemente enthält und diese sich zu Boden senken. 
Flüssiges $\mathrm{Huh}$ u eiwe iss schwärzt Bi nicht (wohl die in demselben enthaltenen flockigen Chalazenreste).

Frische Mileh wirkt im Licht schwach reducirend auf Wismuthsalz, deutlicher als im Uhrglas zeigt sich dies im Reagenzglas an der Oberfläche des Bibodensatzes (andeutungsweise übrigens auch im Dunkeln). Sa re Mileh und Buttermileh wirken ähnlich; g e k o c h t e M i l ch wirkt etwas stärker als rohe.

Sehr zäher grünlicher Schleim aus der Gallenblase schwärzt Wismuth im Licht sehr wenig.

L i n s e n von Ochsenaugen zu Brei zerrieben und mit Bism. subnitr. gemischt schwärzen dasselbe im Licht bis zum folgenden Tage, während die Controlgläser noch nach 4 Tagen weiss sind; ebenso wirkt der Glask örper, soweit er sich mit dem Bismuthbrei mischen lässt.

Durch $\mathrm{R}$ og e n vom Bütt wird Wismuth im Licht deutlich, aber nicht sehr stark geschwärzt (wohl weil nur ein Theil der zahlreichen kleinen Eier sich zerquetschen lässt).

Froschleber (im October untersucht) wirkt im Gegensatz zur Kaninchenleber, auf Bi nicht reducirend.

Auch durch Bierhefe, frisch oder gekocht verwendet, kommt Schwärzung des Wismuthsalzes im Licht nicht zu Stande.

Von Substanzen pflanzlichen Ursprungs wirkte W eizenm e hl und Aleuronatmehl im Licht leicht reducirend, sowohl im rohen wie im gekochten Zustande.

Frischer Kart of felbre i bräunt sich bekanntlich an der Luft durch O-zutritt; dass Licht diesen Vorgang beschleunigte, konnte ich nicht sicher feststellen, ebensowenig, dass beigemengtes Bismuthum subnitricum mit oder ohne Licht dabei verändert wurde:

Auch von frischem A pfel-oder Birnbrei liess sich eine Veränderung des Wismuthsalzes im Licht nicht erkennen.

Sehr stark wurde Wismuth im Licht geschwärzt durch einen Brei von Stapbylococeus aureus; vorheriges Kooben schwächte diese Wirkung erhehlich ab.

Liessen diese Thatsachen den Schluss zu, dass die redueirenden Stoffe nicht dem lebenden oder iiberlebenden Gewebe allein zukommen, sondern dass sie, wenn auch in verminderter Menge im todten Gewebe enthalten seien, so hatte man zu fragen, $a b$ vielleicht bekannte Bestandtheile des Thierkörpers diese Eigenschaft hätten. 
Von den gelösten Eiweisskörpern des Blutserums war die Unwirksamkeit bereits erwiesen, dagegen schien die starke Reduction, welche der Eidotter und zellenreiche Organe (namentlich solche mit jungen Zellen) zeigten, auf Nuclein hinzuweisen.

$\mathrm{Nuclein}$ aus Hefe dargestellt, von $\mathrm{Gr}$ ii ble $\mathrm{r}$ in Leipzig bezogen, wurde mit Kochsalzlösung verrieben oder in verdünntem Alkali gelöst mit etwas Bismuthum subnitricum vermiseht, in $\mathrm{Uhr}$ und Deckglaspräparaten untersucht. Während die dunkel gehaltenen Controlpräparate unverändert blieben, trat an den belichteten nach 1 bis 2 Stunden mebr oder weniger starke Schwärzung ein; der Versuch gelang bei Wiederholung mit demselben Präparat nach längerer Zeit nicht so gut wie Anfangs, auch nicht so gut mit einem später aus derselben Quelle bezogenen Präparat. Die Ursache dieser verschiedenen Resultate vermag ich nicht zu erklären; zı denken wäre eine moleculare Veränderung der Substanz oder an eine Verunreinigung oder eine beim Aufbewahren vor sich gehende Oxydation.

Lecithin erwies sich als vollkommen unwirksam; ebenso Leim.

Ricinusöl, emulgirt oder einfach mit Wismuth verrieben, wirkt ebenso wenig reducirend wie Leberthran. Dagegen wirkt frische Butter im Licht sehr stark reducirend; Rahm wirkt nicht stärker als Milch.

Weiter wurden Kohlenhydrate mit einigen Tropfen Wasser und Wismuthsalz gemischt im Uhrglaspräparat untersucht. Unwirksam war Stärke, roh oder gekocht, Milchzucker, Laevulose, aueh bei Gegenwart von Natron causticum.

Traubenzucker bewirkte einige Male nach mehrstïndiger Besonnung leichte Graufärbung des Wismuthsalzes. Durch Zusatz eines Tropfens Natron causticum wurde die Reduction nicht stärker. Dagegen wirkte sehr deutlich im Licht reducirend G1y cogen, freilich auch nicht immer gleich. Am Besten gelang die Reduction im Licht, wenn man das Glycogen in einigen Tropfen mit Wismuthsalz vermischten Wassers im Uhrglas quellen liess und dasselbe nur mässig verrïhrte; bei vollkommener Lösung, also in sebr verdtinntem Zustande konnte es unwirksam bleiben. Auf 0 haltiges Blut wirkte Glycogen nicht. 
Bevor ich den Versuch mache die mitgetheilten Beobachtungen zu deuten, möchte ich noch über einige andere damit im Zusammenhang stehende Beobachtungen berichten, zunächst iiber die Wirkung farbigen Lichtes.

Ich erreichte dieselbe so, dass ich das wie sonst bergestellte Uhrglaspräparat auf den Boden eines mittelgrossen Becherglases stellte, dieses in ein etwas grösseres mit Flüssigkeit gefülltes Becherglas eintauchte und durch eine Porzellanschale gleichzeitig beschwerte und von anderweitigem Lichte abschloss. Die Verhältnisse waren so gewählt, dass die Flïssigkeit zwischen den beiden Bechergläsern etwa $1 \mathrm{~cm}$ dick war und oben bis an die sich beriihrenden Ränder der beiden Bechergläser reichte.

Als Flïssigkeiten wählte ich 2 schon von Botanikern u. a. verwendete: concentrirte Lösung von Kaliumbichromat und eine nach dem Augenmass concentrirte Lösung von Kupferoxydammoniak. Erstere Flüssigkeit liess in $1 \mathrm{~cm}$ dicker Schicht vom Spectrum nur den Theil zwischen 75 und 54 der $\mathrm{Z}$ e i s s 'schen Scala durch (roth bis griun). Das Kupferoxydammoniak wurde in 2 Concentrationen von mir verwendet, einer tief dunkelblauen, welche nur die rechte Hälfte des Spectrums bis Theilstrich 55, also violetblau und etwas grün, aber in sehr abgeschwächtem Zustande durchliess, während die etwas hellere, noch immer intensiv blaue Lösung in $1 \mathrm{~cm}$ dicker Schicht daneben noch ganz schwaches Licht zwischen 55 und 65 passiren liess. Auf ihre Helligkeit taxirt war diese letztere blaue Flüssigkeit in gleicher Schichtdicke noch immer vielmal dunkler als die Bichromatlösung. Zum Vergleich wurde eine ganz gleiche Gläsercombination, mit destillirtem Wasser gefüllt, aufgestellt.

\section{Versuch XI.}

23. A u g., 9 Uhr. Von frischem Eidotter mit Wismuth vermischt werden ausser dem Controlpräparat für den Dunkelkasten 5 Uhrglaspräparate angefertigt und exponirt, das erste frei, die 4 andern in den beschriebenen Doppelgläsern. - Viel Sonnenschein. 


\begin{tabular}{|c|c|c|c|c|c|c|}
\hline Zeit & $\begin{array}{c}\text { Exposi- } \\
\text { tions- } \\
\text { Dauer }\end{array}$ & 1.. Frei & $\begin{array}{c}\text { Weisses } \\
\text { Licht } \\
\text { 2. Wasser }\end{array}$ & $\begin{array}{l}\text { 3. Kali- } \\
\text { bichromat }\end{array}$ & $\begin{array}{l}\text { 4. Dunkel- } \\
\text { blau }\end{array}$ & 5. Mittelblau \\
\hline $12 \mathrm{Uhr}$ & 3 Std. & schwarz & schwarz & $\begin{array}{c}\text { unverän- } \\
\text { dert. }\end{array}$ & $\begin{array}{c}\text { leicht grau- } \\
\text { lich }\end{array}$ & $\begin{array}{c}\text { leicht } \\
\text { schwärzlich }\end{array}$ \\
\hline $6 \mathrm{Uhr}$ & 9 std. & schwarz & schwạrz & $\begin{array}{l}\text { unverän- } \\
\text { dert }\end{array}$ & $\begin{array}{c}\text { leicht } \\
\text { schwäxzlich }\end{array}$ & $\begin{array}{c}\text { dunkel } \\
\text { schwärzlich }\end{array}$ \\
\hline $\begin{array}{r}24 . \mathrm{Aug} \\
8 \mathrm{Uhr}\end{array}$ & $23 \mathrm{Std}$ & schwarz & schwarz: & $\left\{\begin{array}{c}\text { eine Spux } \\
\text { von Grau- } \\
\text { farbung }\end{array}\right.$ & grauschwarz & grauschwarz \\
\hline
\end{tabular}

In der Tabelle ist die Farbe der Uhrglaspräparate angegeben: ob dieselben frei oder durch eine Wasserschicht hindurch von dem weissen Licht getroffen.wurden, machte also keinen merklichen Unterschied. Das "blaue" Licht wirkte viel sehwächer als das weisse, aber ungleich stärker als das "orange $e^{6}$ Licht, das in den ersten Stunden überhaupt keine Wirkung erkennen liess.

Der Versuch wurde mehrmals wiederholt, sowohl mit Eidotter, wie mit Thymus und Wismuth. Jedesmal ergab sich, dass die stärker brechbare Hälfte des Spectrums die Reduction des Wismuth zu Stande kommen liess, während die schwächer brechbare, obwohl für das Auge viel heller, so gut wie wirkungslos war.

Bei den Besonnungsversuchen ist eine gleichzeitige Erwärmung. häufig nicht ganz auszuschliessen gewesen, da man durch Alaunlösung nur die dunklen Wärmestrahlen ausschalten kann. Diese Erwärmung hat aber, wenn iberhaupt, sicher nur geringen Antheil an der Sonnenwirkung gehabt, denn in den Versuchen mit Blut machte diese sich oft schon nach wenigen Minuten geltend, wo die Temperatur nur wenig gestiegen sein konnte, und die Wismuthversuche ergaben bei diffusem Tageslicht, wenn auch langsamer, das gleiche Resultat wie bei Sonne.

A uer'sches Gasglahlicht wirkte auf Wismuth-Eitermischung bei 5 stïndiger Einwirkung nur ganz schwach schwärzend (trotz erheblicher Erwärmung als Nebenwirkung).

Während bei Versuchen über die O-zehrung der Gewebe die Wahl des Oxyhämoglobins selbstverständlich war, 'ist die Wahl des Bismuthum subnitricum eine willkürliche, durch zufällige gelegentliche Beobachtungen bedingte gewesen. Da sich der Körper als brauchbar zeigte, sind nur wenige Verșuche mit anderen Substanzen gemacht worden: Bismuthum oxychlo-

E. Pflüger, Archiv f. Physiologie. Bd. 57 . 
ratum wurde durch Eiter im Sonnenlicht ebenfalls geschwärat, doch nicht ganz so intensiv wie eine Controlprobe mit Bismuthum subnitricum.

Bismuthum subgallicum (Dermatol) blieb unverändert, ebenso frisch gefälltes Kupferoxydhydrat, welches mit Eiter vermischt belichtet wurde.

Beiläufig constatirte ich übrigens, dass die Methämoglobinbildung im Blut bei Zusatz von Kali chloricum durch Sonnenlicht erheblich beschleunigt wird. Frisches Schröpfkopfblut wird mit $1 / 3$ Volumen $5 \%$ Kalichloricumlösung versetzt (so dass es also $1,25 \%$ Kalichloricum enthält). Das besonnte Uhrglaspräparat zeigt nach $\mathbf{1}^{1} / 4$ Stunde beginnende, nach 4 Stunden vollendete Bräunung (mit dem Absorptionsstreif im Roth), während die Farbe des dunkelgehaltenen Controlglases noch unverändert ist.

Ich wende mich zur Besprechung und Deutung der vorstebend mitgetheilten Versuche.

Zunächst haben sie ergeben, dass die $0-z$ ehrung der G e w e b e, die bekanntlich auch nach dem Tode der Thiere längere Zeit fortbesteht (bei Eiter sogar bis zu 20 Tagen), vom Augenblick des Todes $a b$ schnell an Intensität abnimmt und dass sie im Sonnenlichtenergischerals im Dunkeln vorsich geht. Darin liegt eine Ergänzung zu den Versuchsergebnissen von $\mathrm{Molese}$ ot t und $\mathrm{Fubini}$, welche an isolirten Organen von Kalt- und Warmbliitern die $\mathrm{CO}_{2}$-ausscheidung im Licht gesteigert sahen.

Je kürzere Zeit seit dem Tode des Thieres verflossen ist, um so mehr darf man annehmen, dass die chemischen Vorgänge in den Zellen der Organe den im Leben statthabenden gleichen werden; mit der Zeit, mit dem Zerfall der complicirten Moleküle der lebenden Zelle, werden sie immer mehr davon abweichen, sich vereinfachen; eine scharfe Grenze gegenüber den vitalen Vorgängen wird sich nicht ziehen lassen. Wenn wir nun sahen, dass die O-zehrung in den aus dem Körper entfernten Organen durch Belichtung gesteigert wird, so werden wir einen ähnlich oxydationsbefördernden Einfluss des Lichtes auch auf die lebenden Organe annehmen dirfen. Am beweisendsten sind wohl die Versuche mit den flüssigen Geweben, mit frisch entleertem Eiter und mit leukämischem Blut, weil hier kein weiterer Eingriff statt hatte; sie dienen zur Stütze für die Versuche mit zerkleinerten Organen, welche zu dem gleichen Ergebniss führen.

Sehr nahe liegt ja der Gedanke, dass bei der Erzeugung der O-zehrenden Substanzen Bacterien eine Rolle spielen. Gewiss ist 
auch deren Antheil, besonders in den Versuchen längere Zeit nach dem Tode nicht ganz ausznschliessen, dass sie aber nicht das wesentliche sind, ergibt sich wieder aus der grösseren Reductionsfähigkeit der frischen und der verschiedenen Wirksamkeit verschiedener Organe.

Wir werden daher die reducirenden Substanzen im wesentlichen als Produkte der Zellen ansehen dürfen. Eine dieser reducirenden Substanzen scheint das Glyeogen zu sein ; auch das Nuelein gehört möglicher Weise zu ihnen. Dass es in der That mehrere sein müssen, gebt auch daraus hervor, dass die Reductionsenergie gegenüber dem Oxyhämoglobin und dem Wismuthsalz bei den verschiedenen Organen nicht im gleichen Sinne variirt und dass durch Kochen die reducirende Kraft bei einzelnen Organen sehr erheblich, bei anderen nur unwesentlich beeinflusst wird.

Nach allen diesen Darlegungen ist, wie ich glaube, der Schluss gerechtfertigt, dass auch in der lebenden thierischen Zelle die 0xydationsvorgänge durch Belichtung gesteigert”werden. Die thierische Zelle würde sich damit analog der Pflanzenzelle verhalten und die Reaction der Retinaelemente auf Licht würde nichts exceptionelles, sondern nur ein specieller Fall eines allgemeinen Gesetzes sein. Für den empirisch längst angenommenen Einfluss des Lichts auf den Stoffwechsel und das Allgemeinbefinden ist damit ein elementarer Beweis geliefert.

Zu fordern bleibt freilich noch. der Nachweis am intacten lebenden Körper, an nicht getrennten Theilen desselben. Ich versuchte diesen Nachweis zu führen, indem ich an Hand oder Finger die Circulation durch elastische Abschnürung unterbrach und nach $\mathrm{K}$. Vierord $t$ das Verschwinden der Oxyhämoglobinstreifen zeitlich verfolgte, gleichzeitig an belichteten und unbelichteten Fingern derselben Hand. Einen Unterschied konnte ich hier nicht finden. Zweckmässigere Versuchsanordnung und Wahl des Beobachtungsobjects werden wohl ein anderes Resultat ergeben.

\section{Literatur.}

1. L. A uerbach. Ueber die Einwirkung des Lichtes auf befrnchtete Froscheier. Centralbl. f. d. medic. Wissensch. 1870. S; 357.

2. P. B ert. Physiologie comparée de la respiration. Paris 1870. p. 46.

3. Buchner. Ueber die Schutzstoffe des Serums. Berl. klin. Woch. 1892. S. 452.

4. R. Bow les. Ueber den Einfluss der Sonnenstrahlen auf die Haut. Monatsh. f. pract. Dermatol. 1894. Bd. 18. S. 17. 
H. Quincke : Tueber aen Enfliss des Lichtes auf dén Thierkörper.

5. Th. W. Eng e l m a n n. Hermanns Lehrb. d. Physiol. I. S. 370.

- Ueber Licht als Reiz für Protoplasma. Pflügers Arch. Bd. 19 S. 1.

- Ueber Lichtperception niederster Organismer. Ebendas. Bd. 9 S. 387.

- Ueber Lichtsinn und Einfluss des Lichtes auf die Pewegungen nieder. ster Organismen. Ebendas. Bd. 30. S. 95.

- Ueber Bewegung der Zapfen und Pigmentzellen der Netzhaut unter dem Einfluss des Lichts und des Nervensystems. Ebendas. Bd. 35 . S. 498 .

6. F u bin i und $\mathrm{R}$ o n $\mathrm{ch}$ i. Moleschotts Untersuchung zur Naturlehre. 1878. Bd̀. 12. S. 1.

7. Th. Geissler. Zur Frage über die Wirkung des Lichtes auf Bacterien. Centralbl. f. Bacteriol. 1892. Bd. 11. S. 161.

8. V. G r a b e r. Grundlinien zur Erforschung des Helligkeits- und Farbensinns der Thiere. Prag. Leipzig. 1884.

9. L. Graffen berger. Versuche über die Veränderungen, welche der Abschluss des Lichts in der chemischen Zusammensetzung des thierischen Organismus and dessen N-Umsatz hervorruft. Pflügers Arch. 1892. Bd. 53. Si 238 .

10. F. H a m m er. Ueber den Einfluss des Lichtes auf die Hant. Stuttgart 1891.

11. J. L o e b. Ueber kïnstliche Umwandlung positiv heliotropischer Thiere in negativ heliotropische und umgekehrt. Pflüger's Arch. 1893. Bd.55.

12. $\mathrm{M} \mathrm{o}$ les $\mathrm{ch}$ ot $\mathrm{t}$ und $\mathrm{Fu}$ bini. Ueber den Einfluss des gemischten und farbigen Lichtes auf die Ausscheidung der $\mathrm{CO}_{2}$ bei Thieren. Untersuchungen zur Naturlehre. 1879. Bd. 12. S. 266.

13. C. v. Platen. Ueber den Einfluss des Auges auf den thierischen Stoffwechsel. Pflüg ers Archiv. 1875. Bd. 11. 272.

14. L. R a u m. Der gegenwärtige.Stand unserer Kenntnisse über den Einfluss des Lichtes auf Bacterien und auf den thierischen Organismus. Zeitschr. f. Hygiene Bd. VI. S. 312-361. 1889.

15. J. S chickhardt. Ueber die Einwirkung des Sonnenlichts auf den menschlichen Organismus und die Mikroorganismen und die hygienische Bedeatung desselben. Friedreichs Bl. f. ggerichtl. Medicin. Bd. 44. 1893. S. $351-91$ und S. $400-439$.

16. J. B. Schnetzler. Sur l'influence de la lumière sur le développement des larves de grenouilles. Arch. des sciences de la bibliothèque universelle. Genève Nov. 1874.

17. $\mathrm{S} \mathrm{pallanzán} \mathrm{i.} \mathrm{Mémoires} \mathrm{sur} \mathrm{la} \mathrm{respiration.} \mathrm{Genève} \mathrm{1803,} \mathrm{p.} 86$.

18. S peck. Untersuchungen über den Einfluss des Lichtes auf den Stoffwechsel. Arch. f. exper: Patbologie. 1880. Bd. 12. S. 1.

19. N. Usk of t. Einfluss von farbigem Licht auf das Protoplasma des Thierkörpers. Centralbl. f. d. medic. Wissensch. 1879. S. 449.

20. K. Vi er o rd t. Hämoglobinspectrum am lebenden Menschen. Zeitschr. f. Biologie. 1878, XIY. S. 422. 